Research Article

\title{
Molecular cloning and expression profiling of a chalcone synthase gene from hairy root cultures of Scutellaria viscidula Bunge
}

\author{
Wei Lei, Shao-Hu Tang, Ke-Ming Luo and Min Sun \\ Key Laboratory of Eco-environments in Three Gorges Reservoir Region of Ministry of Education, \\ School of Life Science, Southwest University, Chongqing, China.
}

\begin{abstract}
A cDNA encoding chalcone synthase (CHS), the key enzyme in flavonoid biosynthesis, was isolated from hairy root cultures of Scutellaria viscidula Bunge by rapid amplification of cDNA ends (RACE). The full-length cDNA of $S$. viscidula CHS, designated as Svchs (GenBank accession no. EU386767), was 1649 bp with a 1170 bp open reading frame (ORF) that corresponded to a deduced protein of 390 amino acid residues, a calculated molecular mass of $42.56 \mathrm{kDa}$ and a theoretical isoelectric point (pl) of 5.79. Multiple sequence alignments showed that SvCHS shared high homology with $\mathrm{CHS}$ from other plants. Functional analysis in silico indicated that SvCHS was a hydrophilic protein most likely associated with intermediate metabolism. The active sites of the malonyl-CoA binding motif, coumaroyl pocket and cyclization pocket in $\mathrm{CHS}$ of Medicago sativa were also found in SvCHS. Molecular modeling indicated that the secondary structure of SvCHS contained mainly $\alpha$-helixes and random coils. Phylogenetic analysis showed that SvCHS was most closely related to CHS from Scutellaria baicalensis. In agreement with its function as an elicitor-responsive gene, the expression of Svchs was induced and coordinated by methyl jasmonate. To our knowledge, this is the first report to describe the isolation and expression of a gene from S. viscidula.
\end{abstract}

Key words: chalcone synthase gene, methyl jasmonate, molecular cloning, Scutellaria viscidula Bunge.

Received: March 16, 2009; Accepted: December 1, 2009.

\section{Introduction}

Flavonoids are a large group of widespread plant secondary metabolites involved in numerous biological processes such as protection against UV light, flower coloration, defense against pathogens and pollen development (Winkel, 2002). Chalcone synthase (CHS, EC 2.3.1.74) catalyzes the first committed step of the flavonoid biosynthesis pathway in which three acetate residues from malonyl-CoA and $p$-coumaroyl-CoA are condensed to form naringenin chalcone (Stefan and Axel, 2005) (Figure 1). The chs gene has been extensively studied in a variety of plants, including Oryza sativa, Zea mays and Medicago sativa.

Plants of the genus Scutellaria contain several medicinally important flavonoids, including baicalin, baicalein, wogonoside and wogonin, that are extracted mainly from the roots of representative species such as Scutellaria baicalensis Georgi and Scutellaria viscidula Bunge. The medicinal properties of these root extracts are well-known in traditional Chinese medicine and are widely used to treat inflammatory and bacterial diseases in oriental countries

Send correspondence to Min Sun. Key Laboratory of Eco-environments in Three Gorges Reservoir Region of Ministry of Education, School of Life Science, Southwest University, 400715 Chongqing, China. E-mail: yangtse2006@yahoo.com or thdmast@ gmail.com.
(Yamamoto, 1991). Recent studies have shown that Scutellaria flavonoids have antiviral activity against human immune-deficiency virus (HIV-1) and T-cell leukemia virus (HTLV-1) (Kovács et al., 2004), inhibit the growth of breast, hepatocellular, pancreatic, prostatic, urothelial and colon cancer cells in vitro (So et al., 1997; Ikemoto et al., 2000; Ye et al., 2002), and scavenge free-radicals (Shieh et al., 2000). However, little information is available on the enzymes involved in flavonoid biosynthesis in Scutellaria species.

Methyl jasmonate (MeJA), an endogenous phytohormone, is an important signaling agent and potent elicitor (Yukihito et al., 1996) involved in a range of physiological responses with key roles in plant development (Creelman and Mullet, 1997). Although the mechanism of interaction of MeJA with specific receptors in plant cells remains unclear, there has been increasing interest in the pathways involved in MeJA-mediated activation and regulation of genes coding for enzymes involved in plant secondary metabolism (Heidrun et al., 1992). For example, treatment with MeJA stimulates pal (phenylalanine ammonia lyase), chs (chalcone synthase), sts (stilbene synthase) and ubgat (UDP-glucose:flavonoid 7-O-glucosyltransferase) expression in Vitis vinifera L. (Assia et al., 2008), and enhances the antioxidant activity and flavonid content in blackberries (Wang et al., 2008b). 


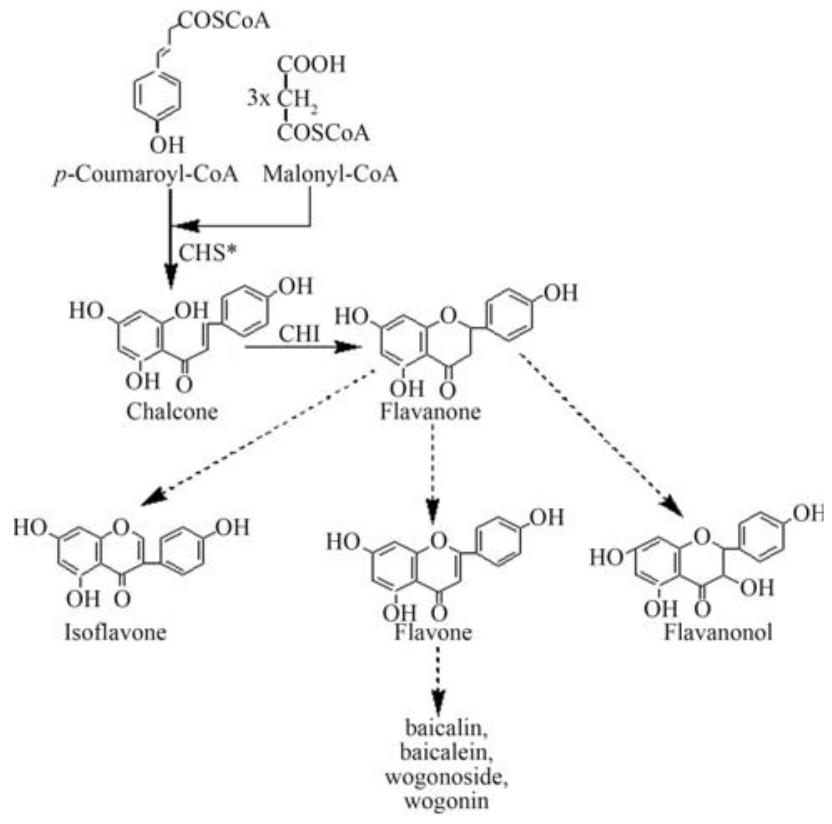

Figure 1 - Flavonoid biosynthetic pathway in Scutellaria cells.

Scutellaria viscidula has a wide distribution in the mountains of Hebei, Shanxi and other provinces in northern China. This species is frequently used as a substitute for the skullcap $S$. baicalensis, for which the demand exceeds supply, primarily because of dwindling natural populations and the difficulty in breeding this species on a commercial scale. The content of medically important flavonoids in S. viscidula has been reported to be higher than in S. baicalensis (Wang et al., 2007). In this report, we provide the first description of the molecular cloning of a chs gene from $S$. viscidula by rapid amplification of cDNA ends (RACE). The expression profile of svchs following induction by MeJA was also investigated. These results broaden our understanding of the structure of the chs gene and the molecular mechanisms involved in flavonoid biosynthesis.

\section{Materials and Methods}

\section{Plant material and treatment with elicitor}

Hairy roots of S. viscidula Bunge were obtained from seedlings and cultivated in $\mathrm{MS}_{0}$ medium (Murashige and Skoog, 1962), as previously described (Wang et al., 2008a). Optimal hairy root lines were subsequently grown in the liquid $1 / 2 \mathrm{MS}_{0}$ medium on a rotary shaker $(110 \mathrm{rpm})$ at $25 \pm 1{ }^{\circ} \mathrm{C}$.

MeJA was added to liquid $1 / 2 \mathrm{MS}_{0}$ medium (final concentration: $200 \mu \mathrm{mol} / \mathrm{L}$ ) and $150 \mathrm{~mL}$ of this solution was placed in $250 \mathrm{~mL}$ conical flasks followed by the inoculation of $\sim 1 \mathrm{~g}$ of fresh hairy roots ( $3 \mathrm{~cm}$ in length). The roots were grown in suspension and after 6,12, 24, 48 and $72 \mathrm{~h}$ samples were obtained to assess chs gene expression, as described below. Appropriate blanks were run in parallel.

\section{Isolation of total RNA}

Total RNA was extracted from S. viscidula shoots and from blank hairy roots with TRIzol reagent according to the manufacturer's instructions (Tiangen, Beijing, China). The quality of RNA were determined by agarose gel electrophoresis and the amount of RNA was quantified spectrophotometrically (DU-640, Beckman, USA). The RNA was stored at $-70{ }^{\circ} \mathrm{C}$ until used.

\section{Cloning of full-length Svchs cDNA by RACE}

Single-strand cDNA was synthesized using TaKaRa RNA PCR kit ver. 3.0 (TaKaRa, Dalian, China) according to the manufacturer's instructions. After RNaseH treatment, the single-stranded cDNA mixture was used as a template for the polymerase chain reaction (PCR). Two oligonucleotide primers (dfchs and drchs, Table S1) were designed based on highly conserved regions in known chs genes from other plant specieses. The PCR reaction was run using the following protocol: initial denaturing at $94{ }^{\circ} \mathrm{C}$ for $3 \mathrm{~min}$, followed by 34 cycles of denaturing for $30 \mathrm{~s}$ at $94^{\circ} \mathrm{C}$, annealing for $30 \mathrm{~s}$ at $55^{\circ} \mathrm{C}$ and extension for $1 \mathrm{~min}$ at $72^{\circ} \mathrm{C}$, with a final extension for $10 \mathrm{~min}$ at $72^{\circ} \mathrm{C}$. After electrophoresis the PCR products were recovered from the agarose gels by using a DNA gel extraction kit (Watsonbiot, Shanghai, China), after which the fragments were ligated into the vector pMD18-T (TaKaRa) and introduced into competent Escherichia coli strain DH5a cells. Recombinant plasmids recovered from positive colonies were sequenced to identify the core fragment.

A SMART RACE cDNA amplification kit (Clontech, USA) was used to isolate Svchs 3'- and 5'-end cDNA. The first-stranded 3'-RACE-ready and 5'-RACE-ready cDNA samples from $S$. viscidula were prepared according to the manufacturer's protocol. A universal primer A mix (UPM, provided in the kit), the 3'-gene-specific primer svchs3-1 (Table S1) and 3'-ready cDNA were used for the first round of 3' RACE. For the nested PCR amplification of 3'RACE, svchs3-2 (Table S1) and nested universal primer A (NUP, provided in the kit) were used.

The 5'-ready cDNA was synthesized with the 5'-CDS primer A and SMART II A oligonucleotide provided in the kit. Two 5'-gene-specific primers, svchs5-1 and svhcs5-2 (Table S1), were designed for 5'-RACE. The first round of PCR amplification was done with primers svchs5-1 and UPM under the following conditions: $1 \mathrm{~min}$ at $94{ }^{\circ} \mathrm{C}, 30 \mathrm{cy}-$ cles of $30 \mathrm{~s}$ at $94^{\circ} \mathrm{C}, 30 \mathrm{~s}$ at $58^{\circ} \mathrm{C}, 2 \mathrm{~min}$ at $72^{\circ} \mathrm{C}$, and finally 10 min at $72^{\circ} \mathrm{C}$. Subsequently, the PCR products were used as templates for nested PCR amplification with the primers svchs5-2 and NUP. The PCR amplification was done using the following conditions: 3 min at $94{ }^{\circ} \mathrm{C}$ followed by $34 \mathrm{cy}-$ cles of $30 \mathrm{~s}$ at $94{ }^{\circ} \mathrm{C}, 30 \mathrm{~s}$ at $55^{\circ} \mathrm{C}$ and $2 \mathrm{~min}$ at $72{ }^{\circ} \mathrm{C}$, and a final $10 \mathrm{~min}$ at $72{ }^{\circ} \mathrm{C}$. The PCR products were purified, subcloned into the vector pMD18-T and transformed into E.coli strain DH5 $\alpha$ followed by sequencing. 
After aligning and assembling the sequences of the core fragment and 3' and 5' RACE ends on ContigExpress (Vector NTI Suite 6.0), the full-length cDNA sequence of svchs was obtained in silico and was used to design a pair of specific primers (fsvchs and rsvchs; Table S1). The fulllength svchs cDNA was subsequently amplified by RTPCR with the specific primer pair using the following conditions: 3 min at $94{ }^{\circ} \mathrm{C}, 34$ cycles of $30 \mathrm{~s}$ at $94{ }^{\circ} \mathrm{C}, 30 \mathrm{~s}$ at $55^{\circ} \mathrm{C}$ and $2 \mathrm{~min}$ at $72^{\circ} \mathrm{C}$, and a final extension for $10 \mathrm{~min}$ at $72^{\circ} \mathrm{C}$ min. The PCR products were purified and cloned into the vector pMD18-T followed by sequencing. PCR amplification and sequencing were repeated three times to confirm the results.

\section{Bioinformatic analysis}

The nucleic acid sequence of the svchs gene (GenBank accession no. EU386767) and corresponding amino acid sequence of the protein ( $\mathrm{SvCHS}$ ) were calculated and analyzed with bioinformatics computer tools. Comparative bioinformatic analysis was done online at the NCBI and Expasy websites. The open reading frame (ORF) was predicted by ORF Finder. Multiple sequence alignments of the amino acid sequences of SvCHS and CHSs from other plant specieses were done with Vector NTI 8.0 using default parameters (Lei et al., 2009). Subcellular location was predicted with the TargetP 1.1 server (Kristin and Siegfried, 2004). Cellular function, transmembrane helices and hydrophobicity of the protein were predicted with the ProtFun 2.2 server (Jensen et al., 2002, 2003), TMHMM server v.2.0 (Ikeda et al., 2002) and ProtScale (Kyte and Doolittle, 1982), respectively. Protein motifs were identified with ScanProsite (Combet et al., 2000). SvCHS and other plant CHS were aligned with ClustalX (Thompson et al., 1997) and subsequently a phylogenetic tree was constructed by the Neighbor-Joining method with 1000 replicates; the reliability of each node was established based on bootstrap calculations using MEGA3 software (Kumar et al., 2001). Homology-based three-dimensional (3D) structural modeling of the SvCHS protein was done with Swiss-Modeling (Arnold et al., 2006) and WebLab ViewerLite 4.2 was used to display the 3D structure.

\section{Expression profile analysis}

To investigate the expression profile of induced svchs, hairy root cultures were treated with MeJA $(200 \mu \mathrm{mol} / \mathrm{L})$ and then harvested after 6, 12, 24, 48 and 72 h. Total RNA was isolated from treated and non-treated hairy roots as already described. The expression profile of svchs was implemented by one-step RT-PCR reactions with $1.0 \mu \mathrm{g}$ of total RNA from each sample, and semiquantitative one-step RT-PCR was done according to the manufacturer's instructions (TaKaRa). All of the RNA templates were digested with RNase-free DNAse I and coding-sequence-specific PCR primer pairs (fexsvchs and rexsvchs) were synthesized (Table S1). PCR amplifications were done in a volume of $25 \mu \mathrm{L}$ using the following conditions: $30 \mathrm{~min}$ at $50{ }^{\circ} \mathrm{C}$ and $2 \mathrm{~min}$ at $94{ }^{\circ} \mathrm{C}$ followed by 25 cycles of $30 \mathrm{~s}$ at $94^{\circ} \mathrm{C}, 30 \mathrm{~s}$ at $55^{\circ} \mathrm{C}$ and $2 \mathrm{~min}$ at $72^{\circ} \mathrm{C}$, and a final extension of $10 \mathrm{~min}$ at $72^{\circ} \mathrm{C}$. The $18 \mathrm{~S}$ rRNA gene was used as an internal control to ensure that equal amounts of total RNA were used in the reactions. This gene was amplified using a one-step RT-PCR reaction and specific primers (18Sf and $18 \mathrm{Sr}$; Table $\mathrm{S} 1$ ) from conserved regions of plant $18 \mathrm{~S}$ rRNA. The PCR products were separated on $1 \%$ agarose gels stained with Goldview and the gray density of the target bands was measured using Gel-Pro Analyzer 4.0 (Media Cybernetics, Bethesda, MD, USA).

\section{Results and Discussion}

\section{Cloning of the full-length cDNA of Svchs}

Oligonucleotide primer pairs for amplification of the $S$. viscidula chs gene were designed based on the conserved regions of known chs genes from other plant specieses $(S$. baicalensis chs-C, S. baicalensis chs-P, S. baicalensis chs and Perilla frutescens chs). A $597 \mathrm{bp}$ cDNA fragment was amplified by RT-PCR and showed $95 \%, 82 \%$ and $82 \%$ sequence similarity with chs genes from $S$. baicalensis, Torenia hybrida and Anthurium andraeanum, respectively, indicating that conserved sequences of the putative svchs gene had been successfully isolated from $S$. viscidula.

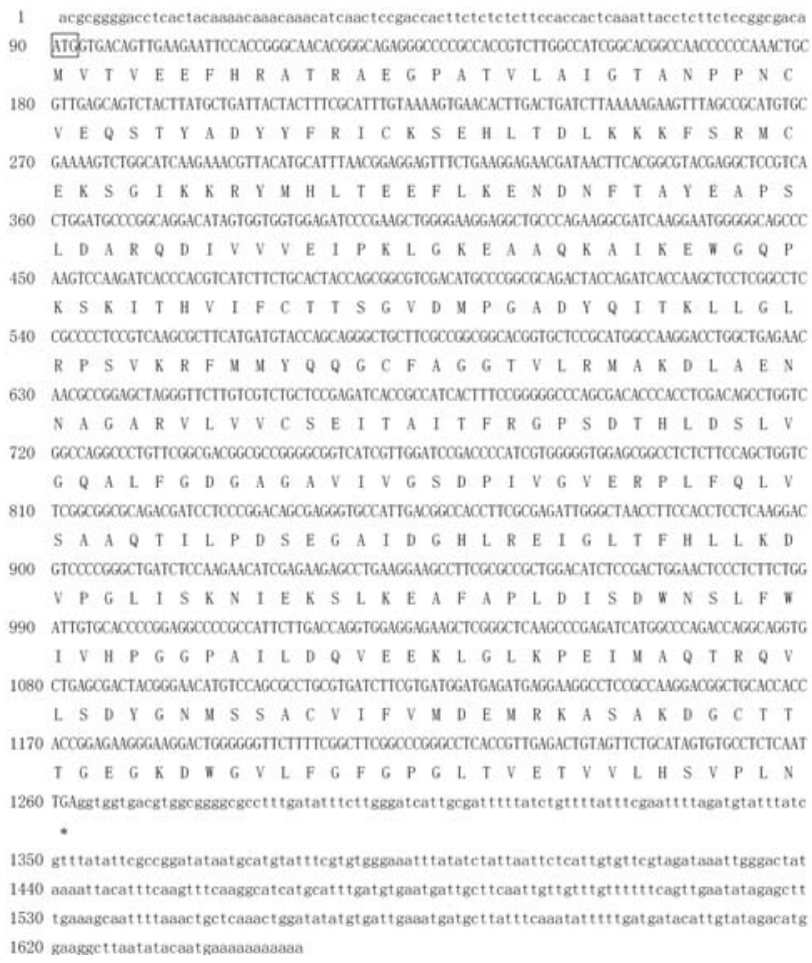

1 acgegsggaceteactacasaacasacaaacateaact cegaceact tetetetet tecaccacteaaat tacetettetecggegace

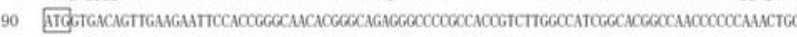
M $V$ V T V V E E 180 GTTGGCAGTCTACTTATGCTGATIACTACTTTCGCATTIGTAMAGTGACACTTGACTGATCTTAMAGGATTTAGCOCCATGTGC V E Q S T Y A D Y Y F R I C K S E H L T D L. K K K K F S R M C

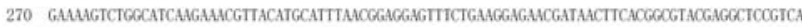
E K S G I K K R Y H H L T E E F L K E N D N F T A Y E A P S 360 CTGGATGOOOGGCAGGACATAGTGGTGGTGGAGATOOCGHAGCTGGGAAGGGAGGCTGOCCAGAAGGCGATCAAGGAATGGCEGCAGCCO

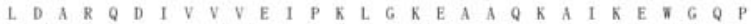
450 AMGTOCANGATCAOCCACGTCATCTTCTCCACTAOCAGCOGOGTCGNCATCOCOGGCGCAGACTAOCAGATCNOCAMCTOCTCGGCCTC

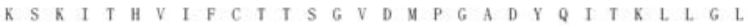

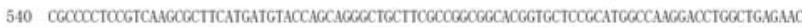
R P S V K R F M I Y Q Q G C F A G G T V L R H A K D L A E N 630 AACOCOCGANCCTMGGGITCTIGTOGTCTGCTOOGAGATCACCGCCATCACTITCCGGGGGCOCAGOGACACOCAOCTOGACNGCCTGGTC N A G A R V L L V V V C S E I I T A I T T F R G P S D T H L D D S L V V 720 GGCCAGCOCCTGTTCGGCGACGGCGCOCGGGOGGTCATOGTTGGATCOGACCOCATCGTGGGGGTGGAGOCGOCTCTCTTCCAGCTGGTC G Q A A L F F G

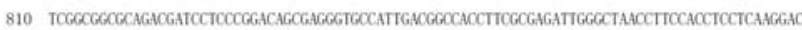
S A A Q T I L P D S E G A I D G H L R E I G L T F H L L K D 900 GTOCOOCGSCTGATCTCCAAGAACATCGAGAGACOCTGAGGGAGCCTTCGCCOCGCTGGACATCTCOGACTOEAACTOCCTCTICTGG V P G L L I S K K N I E K K S L L K E A F F A P L D I I S D W N S L F \#

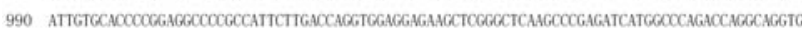
I V 1080 CTGACOGACTMCGGGMCATGTCCAGCGOCTGCGTGATCTTCGTGATOGATGAGATGAGGAGGCCTCOCOCANGGAOCGCTGCAOCACC L. S D Y G N N S S A C V I F V N D E N R K A S A K D G C T T 1170 ACOCGAGMGGGAMGGATGGGGGGTTCTTTCOCCTTCOGCCOGGGCCTCACOGTTGAGACTGTAGTTCTCCATAGTGTGOCTCTCAAT T G E G K D NG V L F G F G P G L T V V E T V V L H S V P L N 1260 TGAkgtggtgacgtg8c Bg8gegectttgatatttet tgggatcatt gcgatttt tatetgtttattegaattttagatgtatttate •

1350 gtttatattegecggatataat geatgtattegtgtgggaaatt tatatctattaattetcattgtgttegtagataaat tgggactat 1440 aaaat tacat tcaagt ttcaaggcatcat gcat ttgat gtgaatgattgct tcaat tgt gtt gt ttttcagttgaatatagagctt 1530 tgaaagcaat tttaaact getcaaactggatatatgtgat gaastgatgettatttcaaatatttt gatgatacat gtatagacatg 1620 gaagret taatatacaatgaaaaaaaaaa

Figure 2 - Full-length cDNA sequence and deduced amino acid sequence of SvCHS. The start (ATG) was boxed and the stop (TGA) codons was marked with asterisks, respectively. The coding sequence of $S v c h s$ is show in capital letters and the 5' - and 3'-untranslated regions are shown in normal letters. 
Gene-specific primers were subsequently designed based on the internal sequence of the core fragment and used to amplify 3'-end and 5'-end cDNA. A 3'-RACE product of $\sim 531$ bp and a $5^{\prime}$-RACE product of $\sim 632$ bp were isolated and sequenced. Finally, the full-length cDNA of svchs was deduced by aligning and assembling the sequences of the core fragment and the 5'-RACE and 3'-RACE ends, followed by confirmation via RT-PCR using a pair of additional full-length cDNA primers. Sequence analysis revealed that the cloned full-length cDNA of the svchs gene (GenBank accession no. EU386767) was 1649 bp long and contained a $1170 \mathrm{bp}$ ORF that encoded a 390 amino acid protein; the gene was flanked by an $89 \mathrm{bp} 5$ '-untranslated region (UTR) and a $390 \mathrm{bp} \mathrm{3'-UTR} \mathrm{with} \mathrm{a} \mathrm{putative} \mathrm{poly-}$ adenylation signal AATAA at a position $100 \mathrm{bp}$ downstream from the stop codon (Figure 2).

\section{Structural characterization and physicochemical properties}

The protein coded by the svchs gene had a calculated molecular mass of $42.56 \mathrm{kDa}$ and a theoretical isoelectric point of 5.79. A BLASTP comparison of the deduced

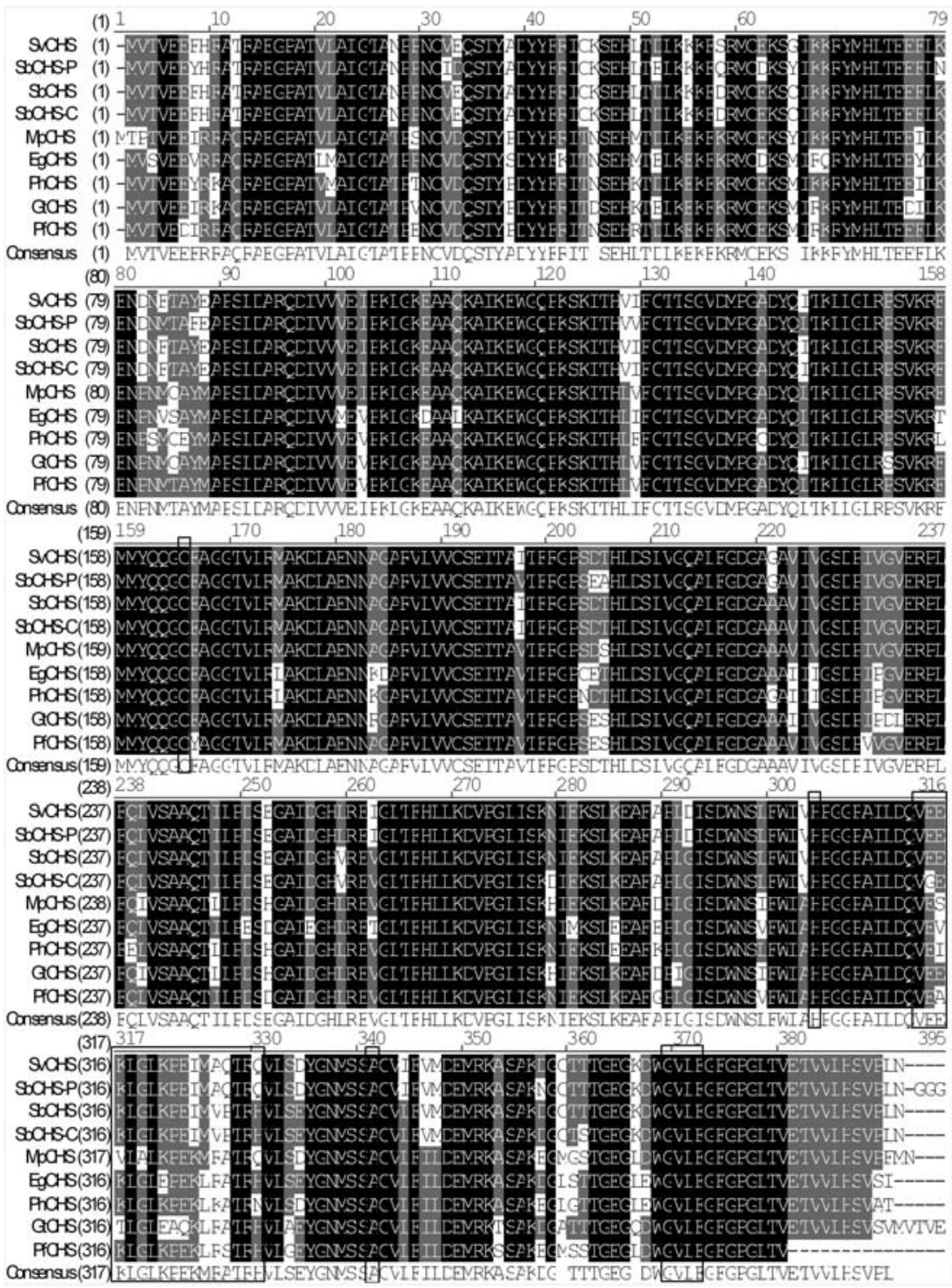

Figure 3 - Multiple sequence alignment of SvCHS and other plant CHS proteins. Identical sites are shown in white letters on a black background, conserved sites are shown in white letters on a gray background and other sites are shown in black letters on a white background. The highly conserved catalytic domain motif Cys-His-Asn, an N-myristoylation motif gvlfgf and a malonyl-CoA binding motif veeklglkpeimaqtrq are boxed. The database accession numbers of the sequences used in the alignment are: Eustoma grandiflorum (EgCHS, GenPept accession no. AB078953), Gentiana triflora (GtCHS, GenPept accession no. D38043), Mazus pumilus (MpCHS, GenPept accession no. AY131328), Perilla frutescens (PfCHS, GenPept accession no. AB002582), Petunia $x$ hybrida (PhCHS, GenPept accession no. X14591), Scutellaria baicalensis (SbCHS-C, GenPept accession no. AB046666), Scutellaria baicalensis (SbCHS-P, GenPept accession no. AF035622), Scutellaria baicalensis (SbCHS, GenPept accession no. AB008748) and Scutellaria viscidula (SvCHS, GenPept accession no. EU386767). 
amino acid sequence of SvCHS with that of other CHS proteins revealed high homology between SvCHS and the corresponding proteins of plants such as Antirrhinum majus ( $88 \%$ identity, $95 \%$ positivity), Misopates orontium (87\% identity, 94\% positivity), Perilla frutescens (87\% identity, 94\% positivity) and S. baicalensis (CHS: 97\% identity, 98\% positivity; CHS-P: $95 \%$ identity, $98 \%$ positivity; CHS-C: $94 \%$ identity, $97 \%$ positivity), indicating that SvCHS belonged to a CHS protein family. Analysis of the subcellular localization by using the TargetP 1.1 server suggested that SvCHS was restricted to the cytoplasm, a finding that agreed with the absence of a signal peptide in the protein and with the demonstration by Hrazdina (1992) that flavonoids are synthesized in the cytoplasm. In addition, the TMHMM Server v.2.0 and ProtScale predicted that SvCHS was a hydrophilic protein with no transmembrane structures; this observation implied that SvCHS catalyzes the biosynthesis of narigenin directly in the cytoplasm, without protein sorting.

Full-length sequence alignment with Vector NTI 8.0 showed that SvCHS has three highly conserved residues $\left(\mathrm{Cys}_{164}, \mathrm{His}_{304}\right.$ and $\mathrm{Asn}_{340}$; Figure 3 ) that form the active center of the CHS catalytic domains (Jez and Noel, 2000). PROSITE predicted that SvCHS contained a binding site (positions 313-330) for malonyl-CoA, a precursor that is cyclized to produce chalcone. In addition, a N-myristoylation site was found at positions 368-373, and myristoylated residues may bind to a hydrophobic core and stabilize the protein structure. The presence of these conserved amino acid residues in the aligned sequences suggested that SvCHS could catalyze the synthesis of chalcone in $S$. viscidula.

\section{Phylogenetic analysis}

chs gene sequences of a variety of plants and bacteria have been reported, with considerable attention being given to the genetic engineering of flavonoids. To examine the phylogenetic relationships among chs genes (Jiang and Cao, 2007), the chs genes of nine plants were aligned (Figure 4). As expected, S. viscidula was closely related to $S$. baicalensis since both belong to the same genus; the relationship observed with the remaining chs genes indicated that this gene encoded a highly conserved enzyme involved in flavonoid biosynthesis.

\section{Three-dimensional model of SvCHS}

Homology-based 3-D structural modeling of SvCHS was done using the program SWISS-MODEL based on the crystal structure of Medicago sativa CHS and subsequently displayed with WelLab ViewerLite. As shown in Figure 5, the secondary structure of SvCHS consisted of random coils, $\alpha$-helixes and extended strands, with the former two being the main components $(43.6 \%$ and $37.4 \%$, respectively). Most of the recognized motifs occurred in the coiled-coil structure of SvCHS, in agreement with the fact that coiled-coils generally contain most of the significant motifs associated with biological functions in a variety of proteins. This analysis indicated that SvCHS had the typical molecular structure of CHS in general and compared fa-

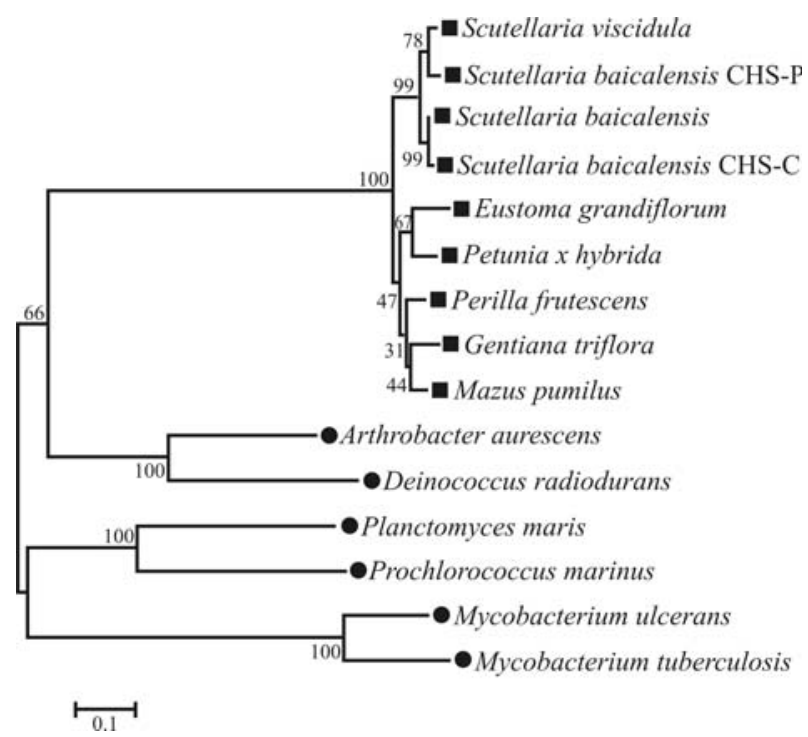

Figure 4 - Phylogenetic analysis of plant and bacterial CHS. The phylogenetic tree was constructed by the Neighbor-Joining method (based on 1000 bootstrap replicates) using MEGA3 software; the bootstrap values are shown on the branches. Plant species are indicated by $(\mathbf{\square})$ and bacterial species by $(\bullet)$. The database accession numbers of the sequences used in the alignment are: Arthrobacter aurescens (AaCHS, GenPept accession no. NC_008711), Deinococcus radiodurans (DrCHS, GenPept accession no. NC_001264), Mycobacterium tuberculosis (MtCHS, GenPept accession no. CP000717), Mycobacterium ulcerans (MuCHS, GenPept accession no. NC 008611), Planctomyces maris (PmCHS, GenPept accession no. NZ_ABCE01000005) and Prochlorococcus marinus (PmCHS, GenPept accession no. NC 005071); the abbreviations and accession numbers for the other protein sequences are indicated in the legend for Figure 3 .

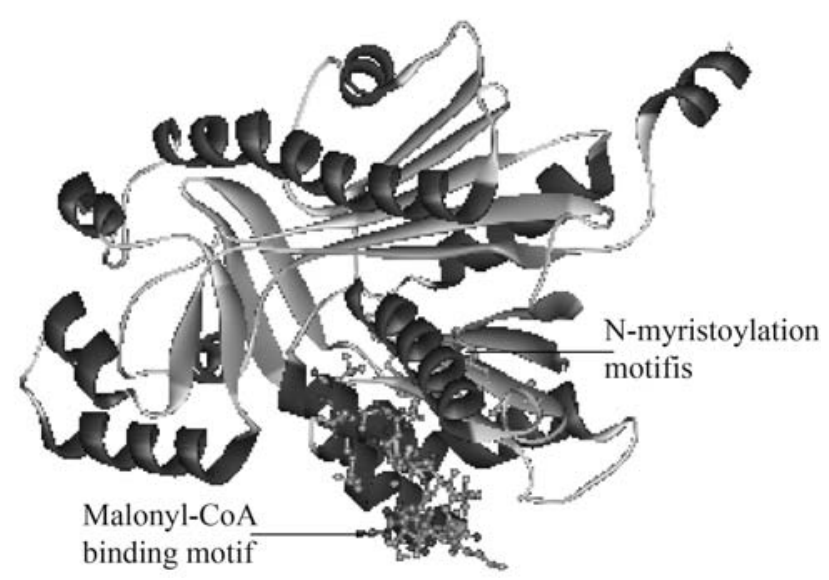

Figure 5 - 3D structural model of SvCHS. The $\alpha$-helix and extended strand are indicated in red and blue, respectively. Random coils are indicated in silver. Selected important motifs are indicated. 


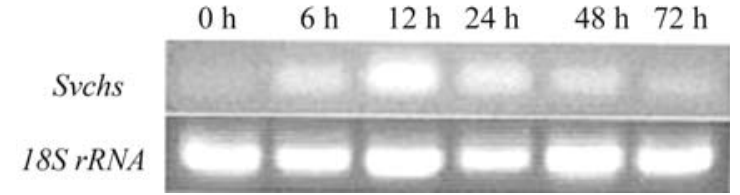

Figure 6 - Expression profile of Svchs following exposure to MeJA for different lengths of time. Upper panel - Svchs expression, lower panel $18 \mathrm{~S}$ rRNA levels used to normalize the amount of template used in the PCR reactions.

vorably with the experimental data for $M$. sativa $\mathrm{CHS}$ (Ferrer et al., 1999; Jez and Noel, 2000).

\section{Expression profile of Svchs following exposure to MeJA}

Short-term exposure to MeJA increases the production of the flavonoid baicalin, whereas long-term exposure inhibits plant cell growth (Zhang and Xu, 2006). We used a one-step RT-PCR with the primers fexsvchs and rexsvchs to investigate the changes in svchs expression following exposure to MeJA. Treatment with MeJA stimulated svchs expression, with the greatest increase occurring after a $12 \mathrm{~h}$ exposure, followed by a progressive decrease in the level of stimulation (Figure 6). This finding indicated that svchs expression was sensitive to MeJA and that the levels of SvCHS may be modulated by this phytohormone. The enhanced expression of svchs in the presence of MeJA would be expected to result in greater CHS activity and the accumulation of plant secondary metabolites in $S$. viscidula, a conclusion in agreement with the observations of Sánchez-Sampedro et al. (2005) that MeJA enhanced CHS activity and increased the levels of silymarin in cell cultures of Silybum marianum.

In summary, we have cloned and characterized an svchs gene from the deciduous medicinal herb $S$. viscidula. The protein encoded by this gene (SvCHS) shares various structural similarities with the corresponding proteins from other plants, especially $S$. baicalensis, including a binding site for malonyl-CoA and an N-myristoylation motif. These structural similarities suggest that SvCHS may be involved in plant intermediate metabolism. Semi-quantitative onestep RT-PCR showed that svchs was responsive to MeJA at the transcriptional level, and this could provide an important mechanism for enhancing intracellular flavonoid levels. These results provided useful information on the induction and regulation of genes involved in flavonoid biosynthesis. Based on the cloning and characterization of this gene, we have constructed a plant expression vector containing svchs that is currently being used to genetically transform $S$. viscidula in order to assess the role of CHS in flavonoid production.

\section{Acknowledgments}

The authors thank Mr. Xichun Pan of the Third Military Medical University (China) for his suggestions. This research was supported by the Key Laboratory of Ecoenvironments in Three Gorges Reservoir Region of Chinese Ministry of Education (grant no. EF200609).

\section{References}

Arnold K, Bordoli L, Kopp J and Schwede T (2006) The SwissModel Workspace: A web-based environment for protein structure homology modeling. Bioinformatics 22:195-201.

Assia B, Nadège T, Cassandrine S, Stéphanie C, Françis B, Saïd H and Mérillon JM (2008) Effect of methyl jasmonate in combination with carbohydrates on gene expression of PR proteins, stilbene and anthocyanin accumulation in grapevine cell cultures. Plant Physiol Biochem 46:493-499.

Combet C, Blanchet C, Geourjon C and Deléage G (2000) NPS@: Network Protein Sequence Analysis. Trends Biochem Sci 3:147-150.

Creelman R and Mullet JE (1997) Biosynthesis and action of jasmonates in plants. Annu Rev Plant Phys 48:355-381.

Ferrer JL, Jez JM, Bowman ME, Dixon RA and Noel JP (1999) Structure of chalcone synthase and the molecular basis of plant polyketide biosynthesis. Nature 8:775-840.

Heidrun G, Martin JM, Toni MK, Kuchan TM and Meinhart HZ (1992) Jasmonic acid is a signal transducer in elicitorinduced plant cell cultures. Proc Natl Acad Sci USA 89:2389-2393.

Hrazdina G (1992) Compartmentation in aromatic metabolism. In: Stafford HA and Ibrahim RK (eds) Recent Advances in Phytochemistry. Plenum Press, New York, pp 1-23.

Ikeda M, Arai M and Lao DM (2002) Transmembrane topology prediction methods: A reassessment and improvement by a consensus method using a dataset of experimentally characterized transmembrane topologies. In Silico Biol 2:1-33.

Ikemoto S, Sugimura K, Yoshida N, Yasumoto R, Wada S, Yamamoto K and Kishimoto T (2000) Antitumor effects of Scutellariae radix and its components baicalein, baicalin, and wogonin on bladder cancer cell lines. J Urol 55:951955.

Jensen JL, Gupta R, Blom N, Devos D, Tamames J, Kesmir C, Nielsen H, Staerfeldt H, Rapacki K and Workman C (2002) Prediction of human orphan protein function from posttranslational modifications and localization features. J Mol Biol 319:1257-1265.

Jensen JL, Stærfeldt HH and Brunak S (2003) Prediction of human protein function according to gene ontology categories. Bioinformatics 19:635-642.

Jez JM and Noel JP (2000) Mechanism of chalcone synthase: pKa of the catalytic cysteine and the role of the conserved histidine in a plant polyketide synthase. Biol Chem 275:3964039643.

Jiang M and Cao JS (2007) Chalcone synthase gene. Chinese J Cell Biol 29:525-529.

Kovács G, Kuzovkina IN, Szõke É and Kursinszki L (2004) HPLC determination of flavonoids in hairy-root cultures of Scutellaria baicalensis Georgi. Chromatographia 60:S81S85.

Kristin E and Siegfried H (2004) InvB is required for type IIIdependent secretion of sopA in Salmonella enterica serovar typhimurium. J Bacteriol 186:1215-1219. 
Kumar SK, Tmamura K, Jakobseni IB and Nei M (2001) MEGA2: Molecular Evolutionary Genetics Analysis software. Bioinformatics 17:1244-1245.

Kyte J and Doolittle RF (1982) A simple method for displaying the hydropathic character of a protein. J Mol Biol 157:105132.

Lei W, Sun M, Luo KM, Shui XR, Sun YM and Tang SH (2009) Computer simulations to characterize chalcone synthase from Scutellaria baicalensis Georgi. Mol Biol 43:10121017.

Murashige T and Skoog K (1962) A revised medium for rapid growth and bioassays with tobacco tissue cultures. Plant Physiol 15:473-479.

Sánchez-Sampedro MA, Fernández-Tárrago $\mathrm{J}$ and Corchete $\mathrm{P}$ (2005) Yeast extract and methyl jasmonate-induced silymarin production in cell cultures of Silybum marianum (L.) Gaertn. J Biotechnol 119:60-69.

Shieh DE, Liu LT and Lin CC (2000) Antioxidant and free radical scavenging effects of baicalein, baicalin and wogonin. Anticancer Res 20:2861-2865.

So FV, Guthrie N, Chambers AF and Carroll KK (1997) Inhibition of proliferation of estrogen receptor-positive MCF-7 human breast cancer cells by flavonoids in the presence and absence of excess estrogen. Cancer Lett 112:127-133.

Stefan M and Axel M (2005) Flavones and flavone synthases. Phytochemistry 66:2399-2407.

Thompson JD, Gibson TJ, Plewniaki F, Jeanmougin F and Higgins DG (1997) The CLUSTAL_X windows interface: Flexible strategies for multiple sequence alignment aided by quality analysis tools. Nucleic Acids Res 25:4876-4882.

Wang SF (2007) Establishment of transform system in Scutellaria viscidula Bunge and its flavonoids production. Master's Thesis, Southwest University, Chongqing.

Wang SF, Sun YM, Lei W, Yang R and Sun M (2008a) Induction and culture of hairy roots in Scutellaria viscidula and its baicalin production. China J Chin Mater Med 33:1669-1672.

Wang SY, Bowman L and Ding M (2008b) Methyl jasmonate enhances antioxidant activity and flavonoid content in black- berries (Rubus sp.) and promotes antiproliferation of human cancer cells. Food Chem 107:1261-1269.

Winkel SB (2002) Biosynthesis of flavonoids and effects of stress. Curr Opin Plant Biol 5:218-223.

Yamamoto H (1991) Biotechnology in agriculture and forestry. In: Bajaj YPS (ed) Medicinal and Aromatic Plants III. Springer Press, Berlin, pp 398-418.

Ye F, Xui L, Yi J, Zhang W and Zhang DY (2002) Anticancer activity of Scutellaria baicalensis and its potential mechanism. J Altern Complem Med 8:567-572.

Yukihito Y, Homare T, Yosuke H and Yasuhiro H (1996) Methyl jasmonate-induced overproduction of paclitaxel and baccatin III in Taxus cell suspension cultures. Nat Biotechnol 14:1129-1132.

Zhang JJ and Xu MJ (2006) Effects of nitric oxide and methyl jasmonate on the baicalin production and cell growth in suspension cultures of Scutellaria baicalensis. Chinese Bull Bot 23:374-379.

\section{Internet Resources}

ORF Finder, http://www.ncbi.nlm.nih.gov/gorf/gorf.html. BLASTP program, http:// www.ncbi.nih.gov/BLAST. SWISS-MODEL, http://swissmodel.expasy.org.

\section{Supplementary material}

The following online material is available for this article:

Table S1. Primers used in molecular cloning and expression profiling of the Svchs gene.

This material is made available as part of the online article available from http://www.scielo.br.gmb.

$$
\text { Associate Editor: Márcio de Castro Silva Filho }
$$

License information: This is an open-access article distributed under the terms of the Creative Commons Attribution License, which permits unrestricted use, distribution, and reproduction in any medium, provided the original work is properly cited. 
Table S1 - Primers used in molecular cloning and expression profiling of the Svchs gene.

\begin{tabular}{ll}
\hline Primers & Sequence \\
\hline dfchs & 5'-TTCATGATGTACCAGCAGGGCTGCT-3' \\
drchs & 5'-GGAGG(A/C)CTTCCTCATCTCATCCA-3' \\
svchs3-1 & 5'-TAACCTTCCACCTCCTCAAGGAC-3' \\
svchs3-2 & 5'-TTCGTGATGGATGAGATGAGGAAG-3' \\
svchs5-1 & 5'-CGTCAATGGCACCCTCGCTGTC-3' \\
svchs5-2 & 5'-TGATGGCGGTGATCTCGGAGCAGA-3' \\
fsvchs & 5'-ACGCGGGGACCTCACTACAAAA-3' \\
rsvchs & 5'-CATTGTATATTAAGCCTTCCATG-3' \\
fexsvchs & 5'-ATGGTGACAGTTGAAGAATTCCA-3' \\
rexsvchs & 5'-ATTGAGAGGCACACTATGCAGAA-3' \\
18Sf & 5'-ATGATAACTCGACGGATCGC-3' \\
18Sr & 5'-CTTGGATGTGGTAGCCGTTT-3' \\
UPM & Long: 5'-CTAATACGACTCACTATAGGGCAAGCAGTGGTATCAACGCAGAGT-3' \\
& Short: 5'-CTAATACGACTCACTATAGGGC-3' \\
NUP & 5'-AAGCAGTGGTATCAACGCAGAGT-3' \\
\hline
\end{tabular}

dfchs - deoxyribonucleotide forward chs, drchs - deoxyribonucleotide reverse chs, svchs - Scutellaria viscidula chs, 3-1, 3-2, 5-1 and 5-2 [3 and 5 refer to 3'end and 5'end, respectively; 1 and 2 refer to the first and the second, respectively], fsvchs - forward svchs, fexsvchs - forward expression svchs, rsvchs - reverse svchs, rexsvchs - reverse expression svchs, NUP - nested universal primer, UPM - universal primer mix, and $18 \mathrm{Sf}$ and $18 \mathrm{Sr}-18 \mathrm{~s} \mathrm{rRNA}$ forward (f) and reverse (r) primers, respectively. 